\title{
On test case generation from asynchronously communicating state machines
}

\author{
O. Henniger \\ GMD-German National Research Center for Information Technology \\ Rheinstr. 75, D-64295 Darmstadt, Germany \\ E-mail: henniger@darmstadt.gmd.de
}

\begin{abstract}
This paper proposes an approach for generating test cases in Concurrent TTCN from a system of asynchronously communicating finite state machines. We give an algorithm for generating a noninterleaving model of prime event structures from a generalized model of asynchronously communicating finite state machines and deal with the generation of test cases from prime event structures.
\end{abstract}

\section{INTRODUCTION}

The behaviour of communicating systems can be modelled by means of a set of finite state machines (FSMs) that run concurrently and communicate with each other via FirstIn-First-Out (FIFO) queues. The formal description techniques Estelle [ISO89] and SDL [ITU92] are based on such a model, extended by additional features.

The behaviour described by an individual state machine is characterized by a set of sequences (i.e. totally ordered sets) of events. The behaviour described by a set of communicating state machines could also be characterized by the set of all possible sequences of events, where events of the individual state machines are intermixed. In fact, this is done in approaches generating a composite state machine and in interleaving semantics definitions. 
Interleaving models preserve many properties of the original specification and are relatively easy to formalize. However, the combinatorial explosion in the number of possible event sequences (interleavings) forms a major problem in generating interleaving models and renders these approaches infeasible in many practical cases. Knowledge of all possible interleavings is in many cases not necessary: If events occurring at different state machines are independent, then their particular order of occurrences does not change their combined effect (cf. e.g. [Pra86]).

Furthermore, interleaving models hide the independence of events occurring at different state machines. This is a problem in test case generation where often a finite subset of possible event sequences has to be selected: In case of independent events from different state machines, there is no way to control the order of occurrence of these events, and there is no guarantee that a selected interleaving can really be observed.

At present, Concurrent TTCN, an amendment to the test description language TTCN [ISO91] designed to specify test cases in a multi-party testing context, is in the process of being standardized. In conventional TTCN, the behaviour descriptions of all test components have to be interleaved in a single tree. Even if split up into several local trees or test steps attached to each other, the behaviour description of a test case forms a single "evaluation tree" as defined in the operational semantics of conventional TTCN. Concurrent TTCN allows for independently executable test components each of which processes its own evaluation tree [BG94].

All these reasons call for the use of noninterleaving models for test case generation and have initiated recent work on this topic. This paper gives an algorithm for generating a prime event structure model from a generalized model of asynchronously communicating state machines. This algorithm is adaptable to the standardized description techniques Estelle and SDL. Furthermore, the paper deals with test case generation from the generated prime event structure.

The rest of the paper is organized as follows: Section 2 briefly reviews related work. Section 3 contains definitions for the models used. Section 4 gives an algorithm for generating a prime event structure equivalent to a set of asynchronously communicating state machines. Section 5 deals with the generation of test cases in Concurrent TTCN from a prime event structure. Section 6 gives concluding remarks.

\section{RELATED WORK}

Previous work on test case generation from state machine models mainly focused on the model of a single FSM (see e.g. [Sid90]) or of a single EFSM (extended finite state machine) ([CA90], [UY91], [CZ93], [HUK95], and others). Test case generation from systems of communicating FSMs requires different approaches. Several approaches for generating black-box test cases from systems of communicating FSMs have been proposed.

Methods with explicit test purposes ([GHN93], [WL93], [FJJV96]) ensure the consistency between test cases, specification, and test purposes and offer much flexibility to the test specifier. However, they require considerable manual effort to define appropriate test purposes and do not guarantee a systematic fault coverage.

Methods with implicit test purposes generally ensure a systematic coverage of the specification. However, as they need to explore the possible behaviour, these methods suffer from the state-explosion problem. Different approaches to alleviate the state-explosion 
problem have been proposed. [LSKP93] pursues an approach similar to program slicing, pruning the given communicating FSMs to contain only a subset of actions; thus yielding a set of smaller, simplified specifications. For synchronously communicating FSMs, [SLU89] and [TK93] generate a composite FSM as an interleaving model by incremental composition and reduction. [AS91], [KCKS96], and [Ulr97] aim at diminishing the state explosion by generating noninterleaving models of the original specification and have inspired the work presented in this paper. [AS91] builds on the reduced reachability analysis approach proposed in [II83] and [KIIY85]. The reduced reachability tree generated by the algorithm presented in [AS91] still contains redundant information. In [KCKS96] a formalized approach is outlined, but no complete algorithm is given. [Ulr97] makes a detour by first transforming the set of communicating FSMs into an 1-safe Petri net, then unfolding the net ([McM95], [ERV96]), and finally constructing a "behaviour machine" as a suitable starting point for test case generation.

We extend the previous work in [AS91], [KCKS96], and [U1r97] by presenting an algorithm for direct transformation of a set of asynchronously communicating state machines into a noninterleaving model suitable for test generation.

The work on test case generation for concurrent systems benefits from the noninterleaving models and methods developed in the context of verification and concurrency theory, such as [Pra86], [Maz88], [Win88], [PL91], [God96], and others.

\section{MODELS}

\subsection{Models for sequential behaviour}

Depending on the level of abstraction, sequential behaviour can be modelled by means of various formalisms such as finite state machines, labelled transition systems, etc. On a high level of abstraction, the behaviour of a sequential discrete event system is characterized by a set of sequences of discrete, observable events, like transmission or reception of messages or time-outs. One distinguishes between events and actions: Actions label events. An event is an occurrence of its action. The same action can be performed various times producing a new, distinguishable event each time.

We use a state machine model similar to the one used in $\left[\mathrm{ZWR}^{+} 80\right]$.

Definition 1 An input-output state machine (IOSM) is a quadruple $\left(S, A, T, s_{0}\right)$ where

- $S$ is a finite non-empty set of states,

- $A$ is a finite non-empty set of actions partitioned into a set of input actions $A_{I}$ and a set of output actions $A_{O}\left(A_{I} \cup A_{O}=A, A_{I} \cap A_{O}=\emptyset\right)$,

- $T \subseteq S \times A \times S$ is the transition relation, and

- $s_{0} \in S$ is the initial state.

An output action is denoted by $! a$; an input action by ?a. Each $t \in T$ is a transition from the present state to a next state, associated either with an input or an output action. An IOSM is represented graphically by a directed graph where nodes represent states and arcs represent transitions. 


\subsection{Models for concurrency}

Various formalisms have been defined for describing the behaviour of concurrent systems. A recent classification is given in [SNW96]. Models for concurrency are classified into:

- behaviour or system models,

- interleaving or noninterleaving models, and

- linear-time or branching-time models.

Behaviour models focus on describing the behaviour in terms of the order of events, abstracting away from states. In contrast, the so-called system models describe the order of events implicitly, explicitly representing states, which possibly repeat. Interleaving models are those that hide the difference between concurrency between several state machines and nondeterminism inside individual state machines. Noninterleaving models take this difference into account. Branching-time models represent the branching structure of the behaviour, i.e. the points in which choices are taken, while linear-time models do not.

\section{Asynchronously communicating input-output state machines}

Communicating state machines can be classified as a system/noninterleaving/branchingtime model. The individual IOSMs communicate with each other and with their environment by exchanging messages. In case of synchronous communication, the exchange of a message between two state machines is regarded as a single event. In case of asynchronous communication, the exchange of a message takes a send and a receive event. After sending a message, the message is buffered for some time, generally in a FIFO queue, before it eventually will be received. We assume asynchronous communication via FIFO queues in order to come close to the semantics of Estelle, SDL, and TTCN.

Different queue semantics can be defined. In Estelle, one can choose whether the messages sent to a state machine (module instance, in Estelle terms) from several other state machines shall be interleaved in a shared queue (common queue) or not (individual queue). In SDL, messages sent to a state machine (process instance, in SDL terms) from arbitrary state machines are always interleaved in a shared queue (input port). In SDL, in some cases communication is nearly synchronous: Any message sent via a signal route or via a nondelaying channel to a process instance waiting in a current state without save or priority inputs is received instantaneously, not needing to be buffered.

We assume a generalized model similar to the one used in [ZWR+80] and base the further discussion on this model. A system of asynchronously communicating IOSMs is composed of a set of IOSMs and a set of perfect (i.e. without loss or reordering of messages) FIFO queues that connect IOSMs with each other and with their environment. Each pair of IOSMs may be connected by at most one FIFO queue for each direction. Note that in contrast to [ZWR ${ }^{+80}$ ] we do not require that the IOSMs form a closed system. We tolerate open interfaces to the environment. This frees us from manually specifying the behaviour of test components, which we would like to find algorithmically. The input messages from the environment are referred to as trigger messages (cf. [AS91]). Figure 1 shows an example system.

\section{Reachability tree}

A reachability tree is a behaviour/interleaving/branching-time model. The reachability tree of a system of asynchronously communicating state machines is a directed tree where 


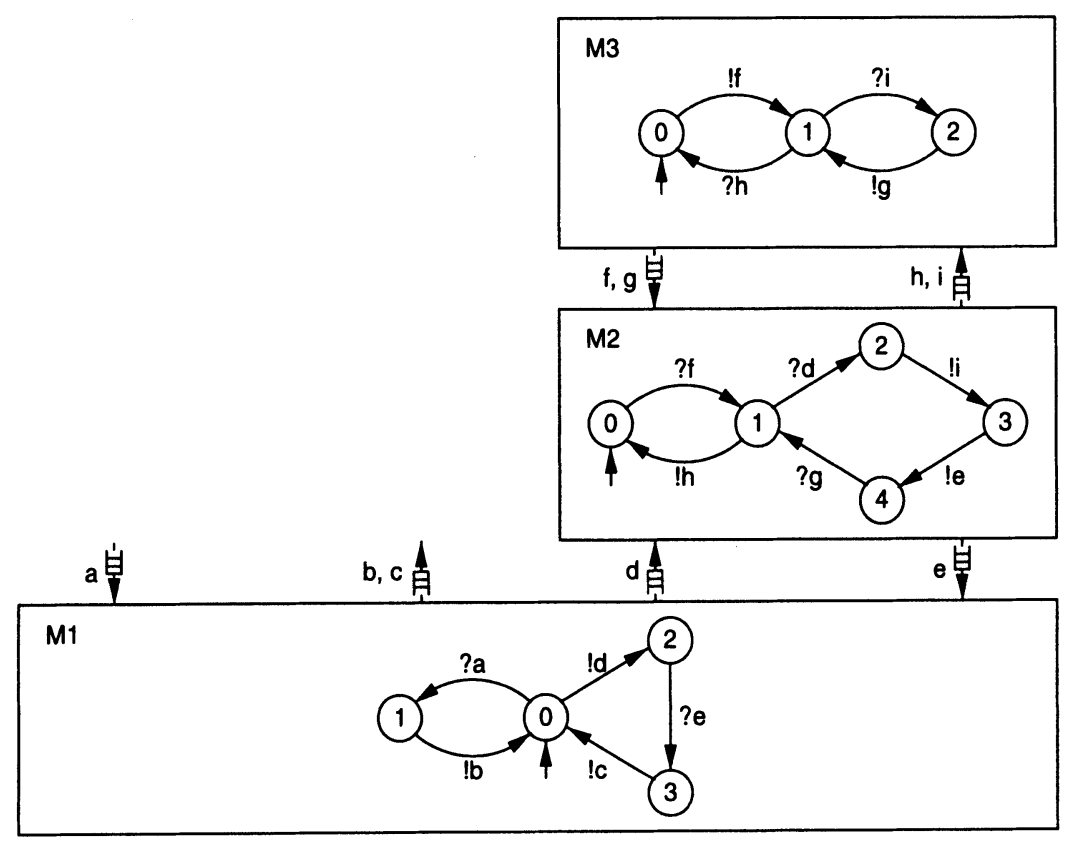

Figure 1 Example 1, a system of asynchronously communicating IOSMs.

the root is the initial global state, the set of nodes is the set of all global states reachable from the initial global state, the set of arcs is a set of events, labelled by actions, and arcs connect subsequent global states.

Figure 2 shows an initial part of the reachability tree for Example 1 computed by the perturbation method [ $\left.\mathrm{ZWR}^{+} 80\right]$. For the generalized model with at most one queue for each direction between each pair of IOSMs, a global state can be nicely represented by means of a matrix $g s$ : Each element $g s_{i i}$ on the diagonal represents the current state of IOSM $m_{i}$, and each off-diagonal element $g s_{i j}, i \neq j$, represents the contents of the queue from $m_{i}$ to $m_{j}$. "-" represents empty and nonexistent queues. The shaded nodes represent global states reached repeatedly, in which the reachability tree has been cut off. The initial global state consists of all IOSMs in their initial states and all queues empty. We assume that a transition with a trigger message (a message from the environment) is always enabled as soon as the IOSM is in the start state of that transition; the environment (which is the tester, in our case) is assumed to place the trigger message at the head of the queue to the IOSM.

The reachability tree models in an interleaving manner the same behaviour as the system of communicating IOSMs. For test case generation, a behaviour model, describing the order of events more explicitly than the compact system model does, would be very helpful. However, the reachability tree overspecifies the order relation and hides the independence of events in separate subsystems. Furthermore, because of the large number of nodes and arcs in the reachability tree (state explosion), computation of the reachability tree of a system of communicating IOSMs is not feasible in most practical cases. In the 


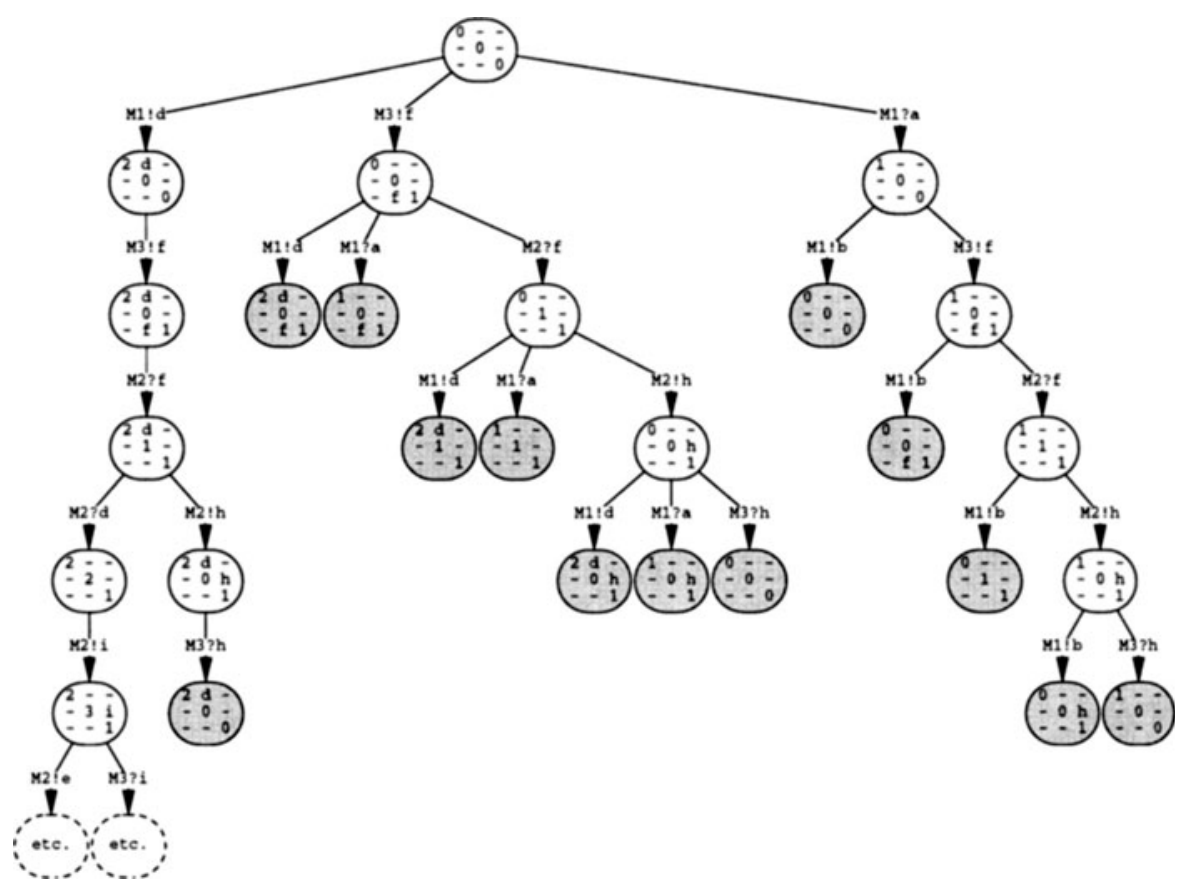

Figure 2 Initial part of the reachability tree for Example 1.

test generation approach described below, the reachability tree will not be computed. Figure 2 has been included to allow a comparison with the noninterleaving behaviour model that we use, the prime event structure (Figure 3 ).

\section{Prime event structure}

Event structures [NPW81] can be classified as behaviour/noninterleaving/branching-time models. Different event structure models have been defined in the literature. An overview can be found in [Kat96].

Definition 2 A (labelled) prime event structure (PES) is a quadruple $(E, \preceq, \#, l)$ where

- $E$ is a countable set of events,

- $\preceq \subseteq E \times E$ is a partial order, the causality relation,

- \# $\subseteq E \times E$ is the (irreflexive and symmetric) conflict relation,

- $l: E \rightarrow A$ is the action-labelling function,

such that $\forall e \in E$ :

(1) $\left\{e^{\prime} \in E \mid e^{\prime} \preceq e\right\}$ is finite, and

(2) $\forall e^{\prime}, e^{\prime \prime} \in E:\left(e \# e^{\prime} \wedge e^{\prime} \preceq e^{\prime \prime}\right) \Rightarrow e \# e^{\prime \prime}$. 


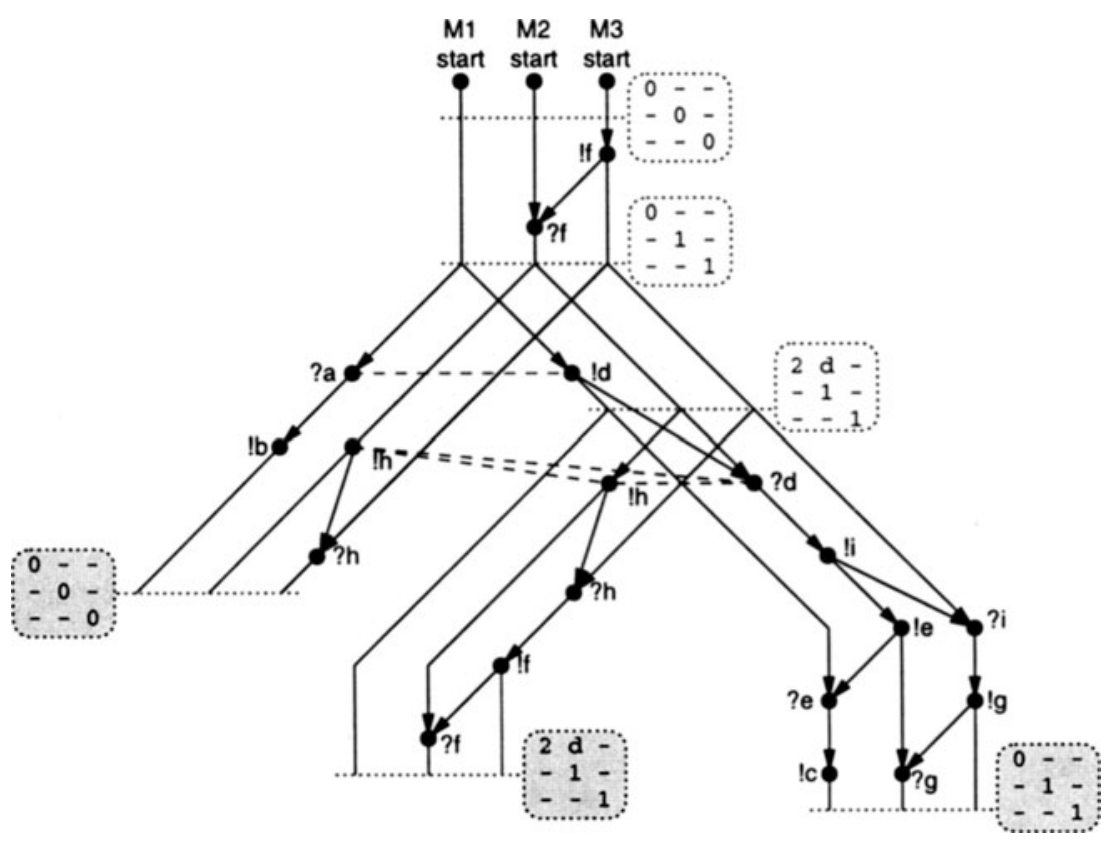

Figure 3 Initial part of the prime event structure for Example 1.

The meaning of $e \preceq e^{\prime}$ is that if the events $e$ and $e^{\prime}$ both happen, then $e$ must happen before $e^{\prime}$. Condition (1) states that the number of causes of any event is finite. The meaning of $e \# e^{\prime}$ is that the events $e$ and $e^{\prime}$ can not happen both. Condition (2) states that if an event $e$ is in conflict with some event $e^{\prime}$, then it is in conflict with all causal successors of $e^{\prime}$ (conflict inheritance property). If two events are neither causally related nor in conflict, these events are independent from each other and both can occur in arbitrary order.

Figure 3 shows an initial part of the PES for the example of Figure 1. A PES is represented as a graph where bold-faced points represent events, directed arcs lead to the immediate causal successors of an event, and undirected dashed arcs connect events in immediate conflict. Next to an event $e$ its label $l(e)$ is indicated. The global states indicated at the margin are not an integral part of the PES. They function as labels indicating where behaviour encountered earlier repeats. Events occurring in the same IOSM form a directed tree. The trees for the concurrent IOSMs are drawn with parallel arcs. The PES resembles the "space-time diagram" introduced in [Lam78], however, not with a linear, but a branching "time axis". 


\section{CONSTRUCTION OF A PRIME EVENT STRUCTURE}

\subsection{Starting point of the test case generation approach}

The starting point of our test case generation approach is a correct specification of the implementation under test (IUT) and of the test context. The test context depends on the chosen test architecture. In the realm of protocol conformance testing, the test context includes the underlying service provider between IUT and lower tester as well as a user above the IUT.

The behaviour of test components needs not to be specified prior to test case generation. It will be derived algorithmically. The test components will be fitted to the open interfaces of the specification of IUT and test context. As for the reachability tree, we assume again that the environment provides for the right trigger messages when they are expected by the specification of IUT and test context. This is an appropriate assumption for conformance testing. It ensures that all expected external behaviour of IUT and test context is covered, while unexpected behaviour (as for robustness tests) is left out.

Correctness of the specification should be checked by validation techniques. In particular, queue overflow is regarded as a potential specification error. Unbounded growth of the number of messages in the queues is a problem in theory and in practice and can be avoided by appropriate design criteria [ $\left.\mathrm{ZWR}^{+} 80\right]$. If all queues are bounded, then the specification has a finite (yet probably very large) state space, and analysis algorithms, like the one described below, terminate.

Figure 1 shows a simple example, applying the remote test method [ISO91]. The IOSM M2 models the IUT. M1 models the service provider between IUT and lower tester. M3 models the user above the IUT. The lower tester will be connected to the open interface of M1. This example is interesting as it is still easy to check, yet contains more than two concurrent IOSMs. As Figure 1 provides for only one test component, we have included another simple example that provides for two test components and is more suitable for demonstrating the generation of test cases in Concurrent TTCN. Figure 4 shows the specification of the same IUT, subjected to the distributed test method [ISO91]. Here, the service access point above the IUT is accessible, and the user above the IUT is replaced by an upper tester. Lower tester and upper tester reside in different real systems and have to communicate with each other using test coordination procedures. Abstract test cases for the distributed test method in conventional TTCN often leave the test coordination procedures unspecified. Abstract test cases in Concurrent TTCN have the advantage that the test coordination procedures are included in terms of coordination messages between the individual test components.

\subsection{Algorithm for constructing a PES}

Below, the algorithm for generating an equivalent PES from a system of asynchronously communicating IOSMs is described in a meta-programming language. To avoid excessive parameter passing, we present the algorithm using global data.

We need some definitions. The global state gs of a system of asynchronously communicating IOSMs is a k-tuple $\left(s_{1}, \ldots, s_{n}, q_{1}, \ldots, q_{m}\right)$ where

- $s_{1}, \ldots, s_{n}$ are the current states of the IOSMs $m_{1}, \ldots, m_{n}$ and

- $q_{1}, \ldots, q_{m}$ are the contents of the queues between the IOSMs. 


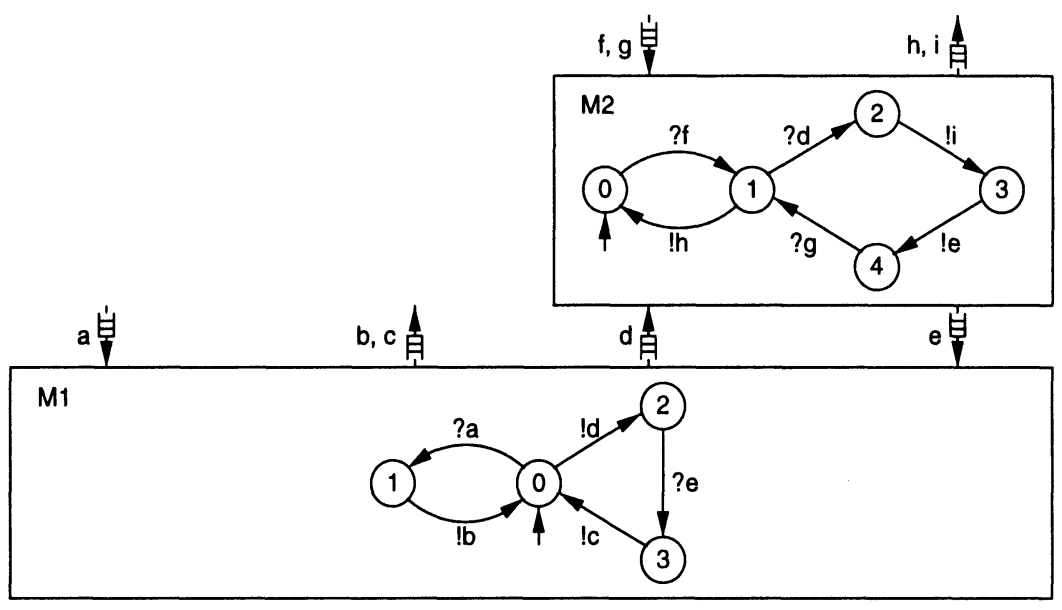

Figure 4 Example 2, a system of asynchronously communicating IOSMs.

A configuration $C$ of a $\operatorname{PES}(E, \preceq, \#, l)$ is a finite subset of $E$ such that:

- $e \in C \Rightarrow \forall e^{\prime} \preceq e: e^{\prime} \in C$ (i.e., $C$ is causally closed), and

- $\forall e, e^{\prime} \in C: \neg\left(e \# e^{\prime}\right)$ (i.e., $C$ is conflict-free).

The final state of a configuration $C$, denoted $f s(C)$, is the global state reached after all events $e \in C$, and no other events, have occurred.

Let $g s$ be the global state of a system of asynchronously communicating IOSMs and let $m_{i}$ be an IOSM from this system. A transition $t=\left(s, \mu, s^{\prime}\right)$ of $m_{i}$ is enabled in $g s$ if the current state of $m_{i}$ is $s$ and either

- $t$ is an output transition $(\mu=! a)$, or

- $t$ is an input transition $(\mu=? a)$ and the message to be received is at the head of the corresponding queue.

$E T_{i}(g s)$ denotes the set of enabled transitions of $m_{i}$ in $g s$. A transition $t=\left(s, \mu, s^{\prime}\right)$ of $m_{i}$ is potentially enabled in $g s$ if the current state of $m_{i}$ is $s$ and $t$ is an input transition $(\mu=? a)$ with the corresponding queue empty. $P T_{i}(g s)$ denotes the set of potentially enabled transitions of $m_{i}$ in $g s$. next $(g s, t)$ denotes the next global state reached from global state $g s$ on execution of transition $t$. The different semantics of Estelle and SDL are taken care of in the generic functions computing the next global state and determining the enabled and potentially enabled transitions.

The algorithm updates $E, \preceq$, and $l$ explicitly. The conflict relation \# is implicitly given as any two events of the same IOSM $m_{i}$ that are not causally related are in conflict to each other. A new event of $m_{i}$ is denoted $e_{i c_{i}}$ where $c_{i}$ is the event counter for $m_{i}$.

The data structure conf represents a configuration of the PES to which events are appended. conf contains the following fields: $f s$, the final state of the configuration; predecessor $_{i}(1 \leq i \leq n)$, the last event for $m_{i}$; send $_{\mu}(\mu \in A)$, a FIFO queue for send 
events with label $\mu$; Wait $_{i}(1 \leq i \leq n)$, the set of potentially enabled transitions that $m_{i}$ waits for. For breadth-first processing of alternative branches, the algorithm uses a FIFO queue data structure conf _queue with the two basic access operations put and get.

The algorithm can be outlined as follows. First, the data structures are initialized. Construction of the PES begins with the initial configuration containing only "dummy" start events, which are needed to make the append procedure applicable also for the beginning of the PES. Each enabled transition that is the only transition of its IOSM in the current state is appended to the PES. The global state reached is added to the set of visited global states Visited. The order of execution does not matter in case that transitions from several IOSMs can be appended. This is repeated until a global state is reached that has been reached before or until no more transitions without alternatives within the same IOSM are enabled. Then, we have reached a point where the PES branches out. For one IOSM $m_{i}$, all enabled alternative transitions are appended to the PES opening up new branches of the PES. If $m_{i}$ has potentially enabled transitions in the current state, an additional new branch is opened up and the potentially enabled transitions are stored in the Wait set. In this new branch, no transitions are appended for $m_{i}$ unless a potentially enabled transition becomes enabled. If the final state of a configuration in a new branch is new, the information in conf about this configuration is put into the FIFO queue conf _queue. One after the other, these configurations will be processed as described above for the initial configuration. This continues until there are no more nodes to be investigated in conf_queue.

Consider the example in Figure 1 and its PES in Figure 3. In the initial global state, the transitions $(0, ? a, 1)$ and $(0, ! d, 2)$ of $\mathrm{M} 1$ and $(0, ! f, 1)$ of $\mathrm{M} 3$ are enabled. $(0, ! f, 1)$ is the only potential transition of M3 in this state and is executed first. In the new global state, the transition $(0, ? f, 1)$, the only potential transition of M2 in this state, is executed. Afterwards, no more transitions without alternatives are enabled, and the two conflicting transitions of M1 are executed leading to two new branches, and so on.

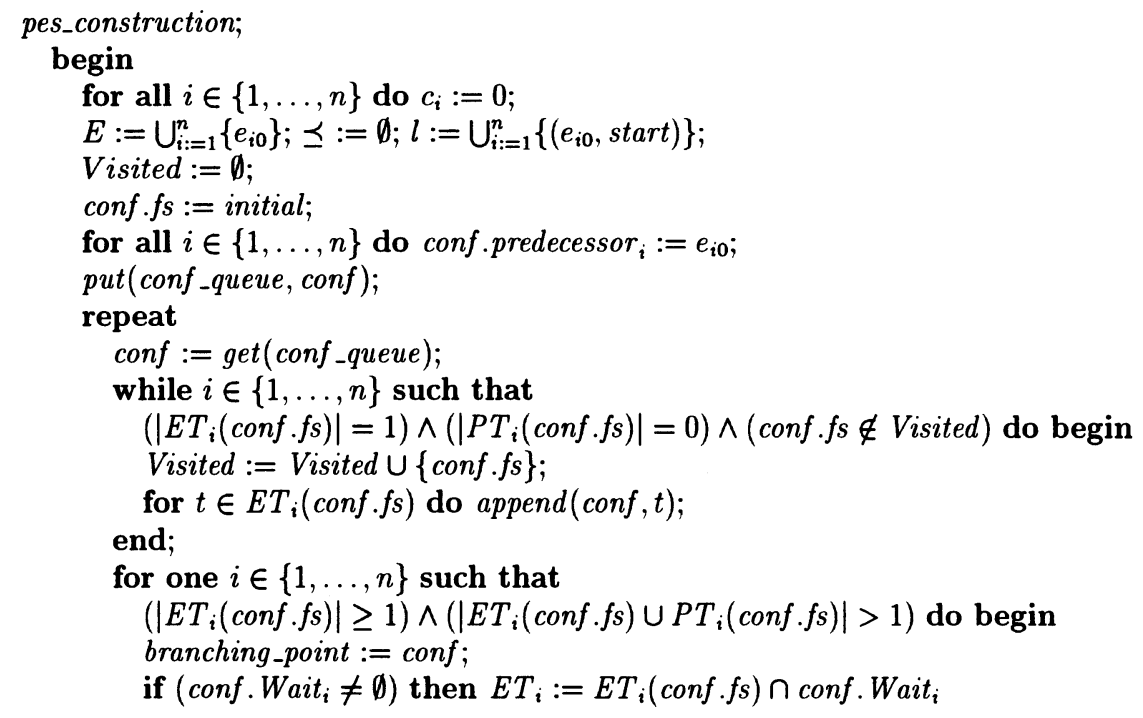




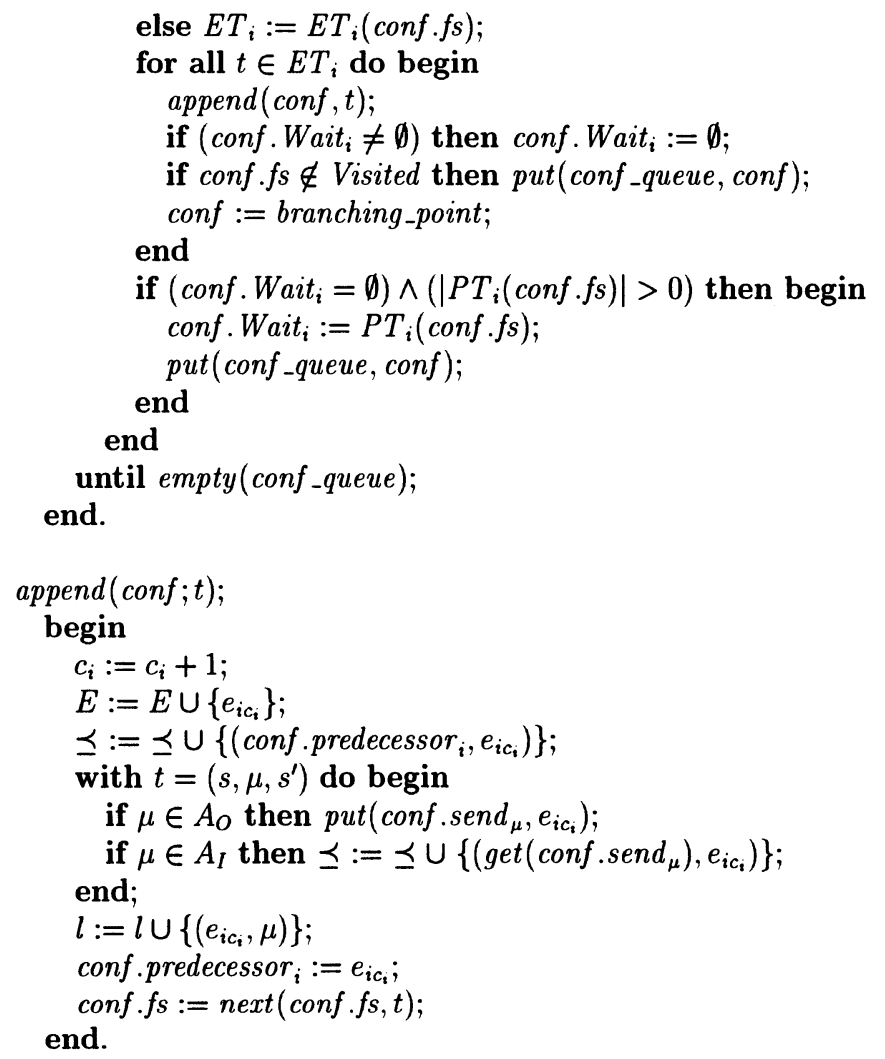

\subsection{Some properties of the generated model}

A discrete event system is in a certain state at any time. The current state of a system depends on which events have happened before, i.e. on the history of the system, and determines which events can happen next, i.e. the possible continuations. In state-oriented models, such as a reachability tree, states of the system are explicitly represented as nodes of the graphical representation. In the PES model, the current global state is not explicitly represented. However, global states of the system are implicitly represented in a distributed manner. Each of the individual IOSMs may be in any "state" along a path of the parallel trees, where all causal predecessors happened before. As an example, consider the left-most path in Figure 3. After performing the event labelled M1? $a$, IOSM M1 may already perform M1! $b$ while M2 is still waiting for M3 to perform M3! $f$ before M2? $f$ may occur. The matrices at the margin of the PES show the final states of some configurations, reached after all events from above the corresponding dotted lines have occurred. They serve as labels to indicate the possible continuations of the PES.

The algorithm generates only an initial part of the PES. The PES is cut off when behaviour encountered earlier repeats. The generated initial part may be expanded by appending the sub-PES's starting with the corresponding global states to the cut-off 


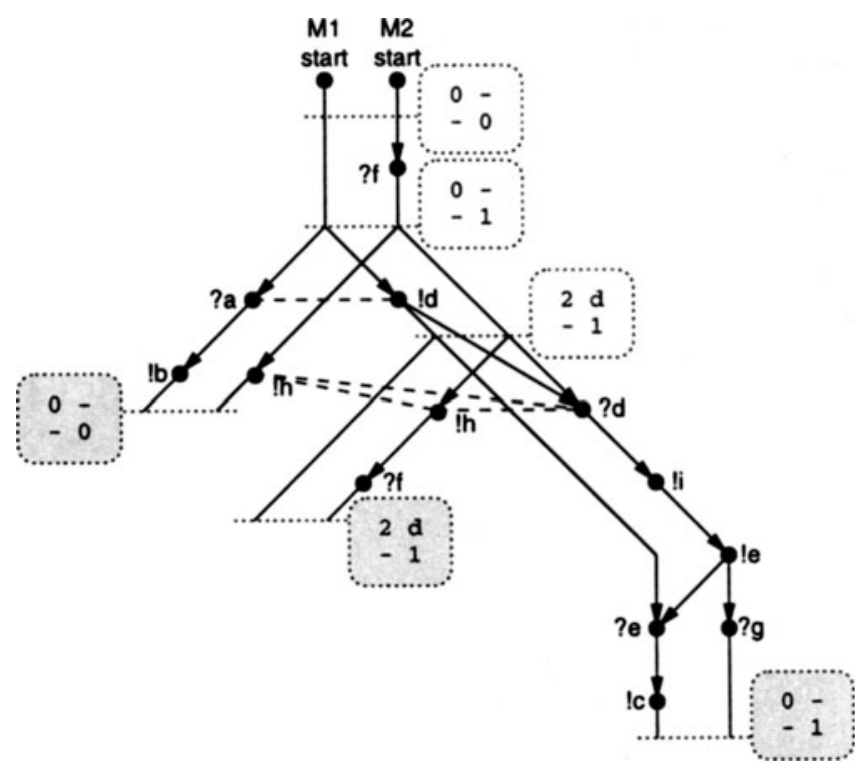

Figure 5 Initial part of the prime event structure for Example 2.

points. A PES $P=(E, \preceq, \#, l)$ of a system of asynchronously communicating IOSMs is complete if for every reachable state $g s$ there exists a configuration $C$ such that:

- $f s(C)=g s$ (i.e., $g s$ is represented in $P$ ), and

- for every transition $\left(s, \mu, s^{\prime}\right)$ enabled in $g s$ there exists a configuration $C^{\prime}=C \cup\{e\}$ such that $e \notin C$ and $e$ is labelled by $\mu$.

The PES obtained by expanding the generated initial part is complete. A proof is omitted here.

A PES does not hide the difference between nondeterminism due to choice of events inside an individual state machine and due to choice of events from different state machines, as interleaving models do. Each branching-point of the PES corresponds to a choice inside an individual state machine. Nondeterminism due to concurrency, i.e. arbitrary order of events occurring at different state machines, does not cause a branching-point in the PES. Paths of the PES represent significantly different behaviour, not only a different order of independent events, as paths of a reachability tree may do.

\section{GENERATION OF TEST CASES IN CONCURRENT TTCN}

As a test case description contains only events occuring at the PCOs of the test architecture (Figure 6), the PES (Figure 5) needs to be restricted to these events (cf. [BGP89]). This is done by labelling events to be deleted by $\tau$, the nonobservable action. Let $A_{P C O}$ 


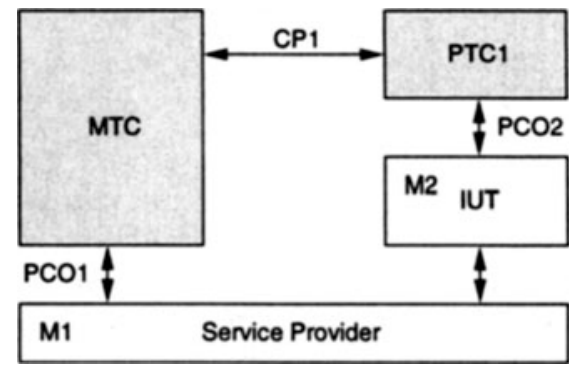

Figure 6 Test architecture for Example 2.

be the set of actions controllable and observable at the PCOs of the test architecture. The projection function is a function $p: A \rightarrow A \backslash A_{P C O} \cup\{\tau\}$ defined by:

$p(\mu)= \begin{cases}\tau & \text { if } a \notin A_{P C O} \\ \mu & \text { otherwise. }\end{cases}$

Application of the projection function to the label of each event of a PES results in an order-preserving mapping to a projection of the PES. In our example, the actions $\mathrm{M} 1 ! d, \mathrm{M} 2 ? d, \mathrm{M} 2 ! e$, and M1?e are not visible at the PCOs and become labelled by $\tau$. The projection can be reduced by skipping events labelled by $\tau$, resulting in a restricted PES (Figure 7). Note that due to the transitivity of the causality relation (as a partial order), there is a directed arc from the event labelled M2! $i$ to the event labelled M1! $c$ in Figure 7 . Due to the conflict inheritance property, there are now dashed arcs between M1? $a$ and $\mathrm{M} 2 ! i$ and between $\mathrm{M} 2 ! h$ and $\mathrm{M} 2 ! i$.

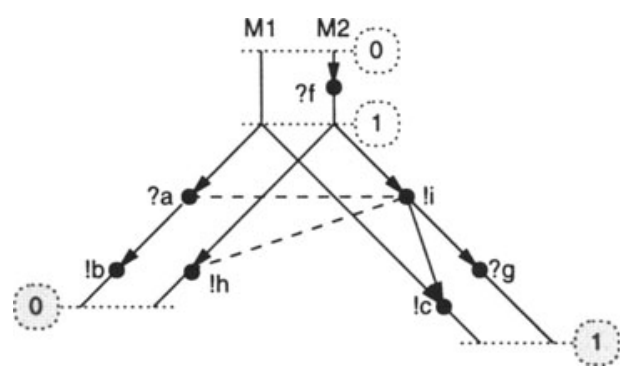

Figure 7 Restricted initial part of the prime event structure for Example 2.

The restricted PES models the behaviour of IUT and test context that is visible at the PCOs. The tester behaviour is the inversion of the restricted PES, i.e. input events are changed to output events and vice-versa. Inversion of inputs and outputs is generally carried out in test generation from asynchronous models.

As each path of the PES represents a significant behaviour, it is desirable that a test suite covers each path of the generated initial part of the PES. We propose to form a test case for each path of the restricted initial part of the PES (hence, a test case generation 
method with implicit test purposes). If the number of paths is too large, an appropriate subset has to be choosen using extra information from outside the specification. Optimization techniques trying to minimize the number of test events are outside the scope of this paper.

As we assume that the tester is, in general, distributed into a main test component and several parallel test components, the events of the inverted restricted PES have to be separated into behaviour descriptions for the individual test components. This step is carried out together with the selection of test cases by traversing a particular path of the inverted restricted PES following the causality relation and recording events belonging to the different test components in separate behaviour trees. If a single test component comprises events from different concurrent IOSMs, then the interleavings of these events have to be computed now. In the behaviour description of a single test component, concurrency can only be expressed by means of interleavings.

If an event $\mu$ of test component $t c_{i}$ is immediately succeeded by an event $\mu^{\prime}$ of another test component $t c_{j}$ (e.g., crossing arrow from M2! $i$ to M1!c in Figure 7) and $\mu$ and $\mu^{\prime}$ are not transmission and reception of the same message, then a coordination message from $t c_{i}$ to $t c_{j}$ is inserted into the test case description. The coordination message informs $t c_{j}$ that $\mu$ has occurred in $t c_{i}$. We use a coordination message if and only if it is necessary in order not to loose sight of the global order of events. We assume that the delay of coordination messages is not larger than the delay of messages in PCOs. Otherwise, one could not tell whether $\mu^{\prime}$ has occurred before $\mu$, which would be wrong behaviour, or after $\mu$, which is correct.

If we reach a branching point in the inverted restricted PES, for each test case one of several conflicting events is selected. All conflicting input events (input to the test component, output from IUT or test context) have to be taken into account in the test case description as alternatives leading to an INCONCLUSIVE verdict. As these events are initiated by IUT or test context, the test components cannot prevent their occurrence though they do not fit to the intended test purpose. If a permissible event occurs that conflicts to the one expected according to the test purpose, one has to assign the INCONCLUSIVE verdict and to try to execute the test case later again.

At the beginning of the behaviour descriptions of the main test component for each test case, CREATE constructs, activating the parallel test components, are inserted. At the end of each path of the initial part of inverted restricted PES, a PASS verdict is assigned. Finally, OTHERWISE events, leading to a FAIL verdict, are added to each level of indentation to deal with any unexpected behaviour. Table 5(a) shows the behaviour description of the test case for the path to the left in Figure 7. Table 5(b) shows the behaviour description for the path to the right.

\section{CONCLUSIONS}

An algorithm for generating a PES equivalent to a system of asynchronously communicating IOSMs has been presented. The algorithm is generic and can be adapted to the semantics of communication over queues used in Estelle and in SDL.

The PES is a suitable starting point for generating test cases in Concurrent TTCN as it specifies the order of events in a noninterleaving manner in a tree structure. How to generate test cases from the PES has been outlined. The approach is applicable for generating multi-party and interoperability test cases. 
Table 1 Test cases for Example 2.

(a)

\begin{tabular}{|ll|}
\hline CREATE(PTC1:PTC1Tree) & \\
PCO1?c & INCONC \\
PCO1?OTHERWISE & FAIL \\
PCO1!a & \\
PCO1?b & PASS \\
PCO1?OTHERWISE & FAIL \\
PTC1Tree & \\
PCO2?OTHERWISE & FAIL \\
PCO2!f & \\
PCO2?h & PASS \\
PCO2?i & INCONC \\
PCO2?OTHERWISE & FAIL \\
\hline
\end{tabular}

(b)

\begin{tabular}{|ll|}
\hline CREATE(PTC1:PTC1Tree) & \\
PCO1?OTHERWISE & FAIL \\
CP1?CM1 & \\
PCO1?c & PASS \\
PCO1?OTHERWISE & FAIL \\
& \\
PTC1Tree & \\
PCO2?OTHERWISE & FAIL \\
PCO2!f & \\
PCO2?i & \\
CP1!CM1 & \\
PCO2!g & PASS \\
PCO2?h & INCONC \\
PCO2?OTHERWISE & FAIL \\
\hline
\end{tabular}

\section{ACKNOWLEDGEMENTS}

The author is grateful to Hasan Ural and Bernd Baumgarten for very helpful discussions.

\section{REFERENCES}

[AS91] N. Arakawa and T. Soneoka. A test case generation method for concurrent programs. In J. Kroon, R.J. Heijink, and E. Brinksma, editors, Protocol Test Systems, IV, pages 95-106, Leidschendam, The Netherlands, 1991. North-Holland.

[BG94] B. Baumgarten and A. Giessler. OSI conformance testing methodology and TTCN. North-Holland, 1994.

[BGP89] B. Baumgarten, A. Giessler, and R. Platten. Test derivation from net models. In J. de Meer, L. Mackert, and W. Effelsberg, editors, Protocol Test Systems, II, pages 141-159, Berlin, Germany, 1989. North-Holland.

[CA90] W. Chun and P.D. Amer. Test case generation for protocols specified in Estelle. In J. Quemada, J. Mañas, and E. Vázquez, editors, Formal Description Techniques, III, pages 191-206, Madrid, Spain, 1990. North-Holland.

[CZ93] S.T. Chanson and J.-S. Zhu. A unified approach to protocol test sequence generation. In INFOCOM '93 [INF93], pages 106-114.

[dBdR88] J.W. de Bakker and W.-P. de Roever, editors. Linear Time, Branching Time, and Partial Order in Logics and Models for Concurrency, Noordwijkerhout, The Netherlands, 1988. Springer-Verlag.

[ERV96] J. Esparza, S. Römer, and W. Vogler. An improvement of McMillan's unfolding algorithm. In T. Margaria and B. Steffen, editors, Tools and Algorithms for the Construction and Analysis of Systems, pages 87-106, Passau, Germany, 1996. Springer.

[FJJV96] J.-C. Fernandez, C. Jard, T. Jéron, and C. Viho. Using on-the-fly verification techniques for the generation of test suites. In R. Alur and T.A. Henzinger, editors, Computer-Aided Verification, 8th International Conference, CAV '96, pages 348359, New Brunswick, NJ, USA, 1996. Springer-Verlag.

[GHN93] J. Grabowski, D. Hogrefe, and R. Nahm. Test case generation with test purpose 
specification by MSCs. In O. Færgemand and A. Sarma, editors, 6th SDL Forum, pages 253-266, Darmstadt, Germany, 1993. North-Holland.

[God96] P. Godefroid. Partial-onder methods for the verification of concurrent systems: an approach to the state-explosion problem. Springer-Verlag, 1996.

[HUK95] O. Henniger, A. Ulrich, and H. König. Transformation of Estelle modules aiming at test case generation. In A.R. Cavalli and S. Budkowski, editors, Protocol Test Systems, VIII, Evry, France, 1995. Chapman \& Hall.

[ICN93] International Conference on Network Protocols, San Francisco, CA, USA, 1993.

[II83] M. Itoh and H. Ichikawa. Protocol verification algorithm using reduced reachability analysis. The Transactions of the IECE of Japan, E 66(2):88-93, 1983.

[INF93] Conference on Computer Communications, IEEE INFOCOM'93, San Francisco, CA, USA, 1993.

[ISO89] Information processing systems - Open Systems Interconnection - Estelle: A formal description technique based on an extended state transition model. International Standard ISO 9074, 1989.

[ISO91] Information technology - Open Systems Interconnection - Conformance testing methodology and framework. International Standard ISO/IEC 9646, 1991.

[ITU92] Specification and description language SDL '92. ITU-T Recommendation Z.100, 1992.

[Kat96] J.-P. Katoen. Quantitative and qualitative extensions of event structures. $\mathrm{PhD}$ thesis, University of Twente, 1996.

[KCKS96] M.C. Kim, S.T. Chanson, S.W. Kang, and J.H. Shin. An approach for testing asynchronous communicating systems. In B. Baumgarten, H.-.J. Burkhardt, and A. Giessler, editors, Testing of Communicating Systems, IX, Darmstadt, Germany, 1996. Chapman \& Hall.

[KIIY85] M. Kajiwara, H. Ichikawa, M. Itoh, and Y. Yoshida. Specification and verification of switching software. IEEE Transactions on Communications, COM-33(3):193-198, March 1985.

[Lam78] L. Lamport. Time, clocks, and the ordering of events in a distributed system. Communications of the ACM, 21(7):558-565, 1978.

[LSKP93] D. Lee, K.K. Sabnani, D.M. Kristol, and S. Paul. Conformance testing of protocols specified as communicating FSMs. In INFOCOM '93 [INF93], pages 115-127.

[Maz88] A. Mazurkiewicz. Basic notions of trace theory. In de Bakker and de Roever [dBdR88], pages 284-363.

[McM95] K.L. McMillan. A technique of state space search based on unfolding. Formal Methods in System Design, 6(1), 1995.

[NPW81] M. Nielsen, G. Plotkin, and G. Winskel. Petri nets, event structures and domains, part I. Theoretical Computer Science, 13:85-108, 1981.

[PL91] D.K. Probst and H.F. Li. Partial-order model checking: a guide for the perplexed. In $\mathrm{K}$. Larsen and A. Skou, editors, Computer-Aided Verification, 3rd International Conference, CAV '91, pages 322-331, Aalborg, Denmark, 1991. Springer-Verlag.

[Pra86] V. Pratt. Modeling concurrency with partial orders. International Journal of Parallel Programming, 15(1):33-71, 1986.

[Sid90] D.P. Sidhu. Protocol testing: the first ten years, the next ten years. In L. Logrippo, R.L. Probert, and H. Ural, editors, Protocol Specification, Testing, and Verification, $X$, pages 47-68, Ottawa, Ontario, Canada, 1990. North-Holland.

[SLU89] K.K. Sabnani, A.M. Lapone, and M.Ü. Uyar. An algorithmic procedure for checking safety properties of protocols. IEEE Transactions on Communications, 37(9), 1989.

[SNW96] V. Sassone, M. Nielsen, and G. Winskel. Models for concurrency: Towards a classification. Theoretical Computer Science, pages 297-348, 1996.

[TK93] K.C. Tai and P.V. Koppol. Hierarchy-based incremental analysis of communication 
protocols. In ICNP '93 [ICN93], pages 318-325.

[Ulr97] A. Ulrich. A description model to support test suite derivation for concurrent systems. In M. Zitterbart, editor, 10. GI/ITG-Fachtagung Kommunikation in Verteilten Systemen, Braunschweig, Germany, 1997.

[UY91] H. Ural and B. Yang. A test sequence selection method for protocol testing. IEEE Transactions on Communications, 39(4):514-523, 1991.

[Win88] G. Winskel. An introduction to event structures. In de Bakker and de Roever [dBdR88], pages 364-397.

[WL93] C.J. Wang and M.T. Liu. Automatic test case generation for Estelle. In ICNP '93 [ICN93], pages 225-232.

[ZWR $\left.{ }^{+} 80\right]$ P. Zafiropulo, C.H. West, H. Rudin, D.D. Cowan, and D. Brand. Towards analyzing and synthesizing protocols. IEEE Transactions on Communications, COM28(4):651-660, April 1980. 